\title{
Numerical simulations of the local generation of internal solitary waves in the Bay of Biscay
}

\author{
N. Grisouard and C. Staquet \\ LEGI, UJF/CNRS/G-INP, BP 53, 38041 Grenoble Cedex 9, France
}

Received: 20 May 2010 - Revised: 31 August 2010 - Accepted: 17 September 2010 - Published: 13 October 2010

\begin{abstract}
Oceanic observations from the Bay of Biscay, Portugal, Mozambique Channel and Mascarene Ridge have provided evidence of the generation of internal solitary waves due to an internal tidal beam impinging on the thermocline from below - a process referred to as "local generation". Here we present two-dimensional numerical simulations with a fully nonlinear nonhydrostatic model of situations that are relevant for the Bay of Biscay in summer. We show that a beam impinging on a thermocline initially at rest can induce a displacement of the isopycnals, large enough for internal solitary waves to be generated. These internal solitary waves however differ from those observed in the Bay of Biscay through their amplitude and distance between wave trains. We then show that the latter feature is recovered when the background flow around the thermocline as found in the Bay of Biscay is included in the forcing, thereby yielding a more accurate view on the local generation mechanism.
\end{abstract}

\section{Introduction}

Internal waves have numerous effects in the ocean. They increase the amount of nutrients that are to be found just under the sea surface, they locally modify the fluid density distribution and therefore modify the ray paths of sound waves with consequences for sonars. They are also believed to have a key role in maintaining the thermal balance and circulation of the ocean by dissipating a significant fraction of the tidal energy that is put into the oceans (Munk and Wunsch, 1998; Wunsch and Ferrari, 2004).

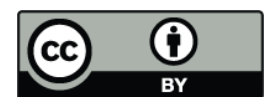

Correspondence to: N. Grisouard (nicolas.grisouard@legi.grenoble-inp.fr)
Internal wave restoring forces are buoyancy and the Coriolis pseudo-force. The dispersion relation of these waves is expressed as (e.g., Gill, 1982, §8.4):

$\omega^{2}=N^{2} \sin ^{2} \theta+f^{2} \cos ^{2} \theta$,

where $\omega$ is the frequency of the waves, $N$ the Brunt-Väisälä frequency, $\theta$ the angle of the wave vector relative to the vertical and $f$ the Coriolis parameter. For a fluid in which only the temperature acts on density, $N$ is defined as $N^{2}=$ $\alpha g\left(d T_{0} / d z\right)$, where $\alpha$ is the thermal expansion coefficient, $g$ the gravity, $T_{0}(z)$ the background temperature profile and $z$ the vertical coordinate oriented positively upwards.

A noticeable feature of Eq. (1) is that $\omega$ is bounded by $\min (N,|f|)$ and $\max (N,|f|)$ (assuming $\omega>0$ ): oscillatory motions are therefore freely propagating within this interval and vertically evanescent outside. In a fluid in which $N$ varies along the vertical, this allows for wave trapping. For instance, a typical stratification in mid- and tropical latitudes is strongly inhomogeneous with an upper mixed layer, an intermediate thermocline supporting high values of $N$ and finally the less stratified and much deeper abyssal ocean. Internal waves of tidal frequency (referred to as the internal tide) are of low enough frequency to propagate down to the bottom of the ocean. The internal tide may encounter a sharp elevation of the bathymetry (continental shelf or sill) and therefore increase in amplitude to preserve the energy flux. Nonlinear transfers of energy to high frequencies then take place. These frequencies are often too high for the associated waves to propagate back in the abyssal ocean but are low enough for these waves to propagate in the thermocline. Strikingly, the subsequent confinement of energy usually leads to a selforganisation of the waves in the form of nonlinear, nonhydrostatic dispersive waves which have been successfully described in terms of internal solitary waves (ISWs), or solitons. For reviews on ISWs in the ocean, see e.g., Ostrovsky and Stepanyants (1989); Helfrich and Melville (2006).

Published by Copernicus Publications on behalf of the European Geosciences Union and the American Geophysical Union. 
However, New and Pingree (1990) observed ISWs in the central Bay of Biscay, too far from the shelf break or any other bathymetry to have been generated there. These authors conjectured that in this case, ISWs are generated by an internal tidal beam (ITB) hitting the seasonal thermocline from below. This ITB originates from the shelf break, with downward propagation of energy into the abyssal ocean, which turns into upward propagation when it reflects from the bottom. On its upward path, the ITB finally encounters the seasonal thermocline, perturbs it locally which may lead to ISWs in the thermocline. New and Pingree coined the term "local generation" of ISWs to refer to this process. Measurements of the ITB location were consistent with ray path computations based upon the local stratification and upon the bathymetry of the Bay of Biscay (Pingree and New, 1991). Further evidence of local generation of ISWs was presented by New and Pingree (1992), giving further support for the existence of local generation. Since the beginning of the 2000s, new remote sensing techniques - namely Synthetic Aperture Radar (SAR) (New and da Silva, 2002; Azevedo et al., 2006) and ocean colour imaging (da Silva et al., 2002) - have confirmed the occurrence of this mechanism in spring and summer, when the seasonal thermocline is present. SAR imagery recently also showed the occurrence of local generation off Southwest Portugal (da Silva et al., 2007) and in the Mozambique Channel (da Silva et al., 2009). This mechanism might also explain observations near the Mascarene Ridge by Konyaev et al. (1995).

The underlying physical mechanism should require very specific conditions to be met, as suggested by the sparsity of the observations. Few attempts have been made so far to explain it quantitatively (see Grisouard et al., 2010, hereafter referred to as GSG10, for a review). The starting point is to derive conditions which maximize the interfacial displacement of the thermocline by the impinging beam, as first done by Delisi and Orlanski (1975). This work was extended by Thorpe (1998) and applied to the Bay of Biscay, to examine whether the local generation process proposed by New and Pingree could occur. This problem was later addressed by Akylas et al. (2007) using asymptotic approaches in the linear and weakly nonlinear, long wave regimes (yielding a soliton equation in the latter case). Gerkema (2001) and Maugé and Gerkema (2008), considering an abyssal ocean of finite depth, solved the problem analytically in terms of vertical modes. Although these approaches varied in numerous aspects, they all relied on the comparison between the phase speed of the interfacial waves in the equivalent twolayer system (i.e. for an equivalent homogeneous abyssal ocean) and the dominant phase speed of the forcing wave. The forcing is found optimal when the ratio of these two waves is of order one (Delisi and Orlanski, 1975; Thorpe, 1998; Akylas et al., 2007), a case referred to as "moderate" by Gerkema (2001). From a qualitative point of view, an optimal forcing promotes the nonlinear steepening of the interfacial waves, thereby preventing internal wave radiation in the abyssal ocean. Moreover, as first noticed by Gerkema (2001), when this condition is met, the ITB experiences multiple reflections and transmissions and therefore scattering. When this condition is not met, the ITB is either completely transmitted through the thermocline (the interfacial phase speed being slower than the forcing wave) or completely reflected against it (the interfacial phase speed being faster than the forcing wave).

GSG10 successfully modelled the local generation process by allowing fully nonlinear and nonhydrostatic effects (as the authors used the term "pycnocline" rather than "thermocline", we will use this terminology when referring to their work). The set-up was academic, namely two-dimensional, non-rotating with idealised forcing and stratification. The stratification profile was again composed of three parts: an upper homogenous layer, a lower deep layer with constant Brunt-Väisälä frequency $N_{0}$ and a pycnocline in-between. The difference with most previous works was that the density variation across the density jump was continuous with a pycnocline of finite thickness. By varying the amplitude of the density jump across the pycnocline and the horizontal phase speed of the forcing beam, GSG10 derived conditions to generate mode- $n$ ISWs, showing the crucial importance of the finite thickness of the pycnocline. They applied these conditions to a typical stratification of the Bay of Biscay in summer when local generation is observed. Estimating the horizontal phase speed of the beam from Gerkema et al. (2004) numerical data, they concluded that both mode- 1 and mode-2 ISWs could be generated, without being able to anticipate the dominant mode. Observations show that mode-1 ISWs are generated in the Bay of Biscay. An agreement with these observations was therefore possible but further investigations were required. This is the purpose of the present paper.

Following GSG10, we present a new set of numerical experiments performed with the MIT general circulation model, of which parameters are directly based on those of the Bay of Biscay in summer. The numerical set-up is described in Sect. 2. In Sect. 3, we consider the effects of the impact of the ITB on a thermocline initially at rest, its subsequent scattering and the generation of ISWs. Our goal is to verify whether local generation occurs with these parameters and whether the dominant mode number, if any, agrees with the predictions of GSG10. We show that these predictions are confirmed but the ISWs generated by this process are noticeably different from those observed in the Bay of Biscay. The trains of ISWs are separated by 25 to $30 \mathrm{~km}$ (in agreement with the predictions of GSG10), whereas in the Bay of Biscay this distance is about $50 \mathrm{~km}$. We next point out that, regardless of the location at which the ITB impinges on the thermocline, the internal tide in the Bay of Biscay is dominated in the upper $100 \mathrm{~m}$ by a signal of horizontal wavelength $50 \mathrm{~km}$. This similarity suggests that this signal may also play a role in the local generation process in the Bay of Biscay. To support this idea, we carry out in Sect. 4 two simulations 
(a)

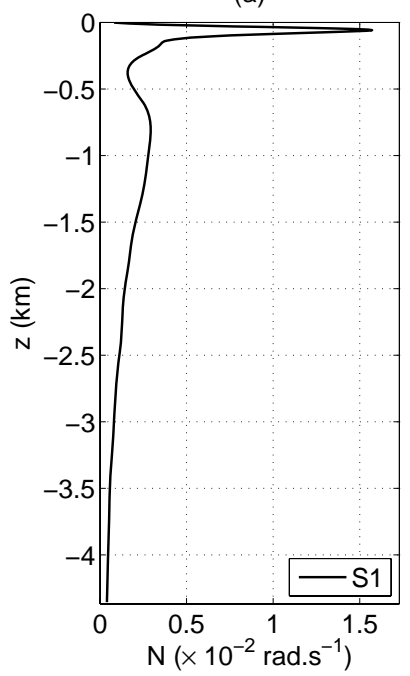

(b)

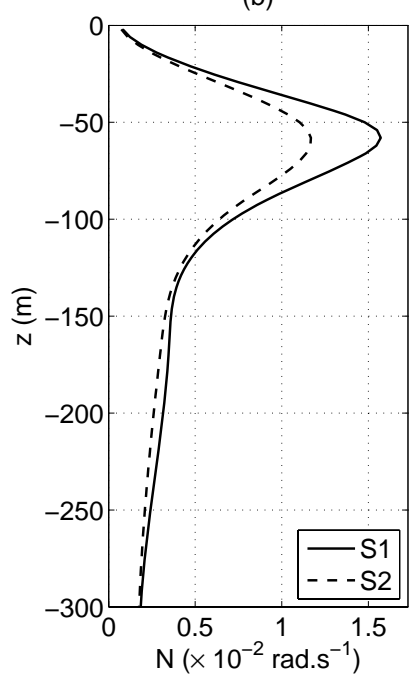

Fig. 1. (a): Brunt-Väisälä profile for stratification $S 1$ over the whole water depth $(4370 \mathrm{~m})$. (b): Brunt-Väisälä profiles for stratifications $\mathrm{S} 1$ and $\mathrm{S} 2$ from 0 to $300 \mathrm{~m}$ depth. Below $300 \mathrm{~m}$ depth, S1 and S2 are identical.

with a forcing including both components of the internal tide. Clear trains of mode-1 ISWs are now generated, separated by $55 \mathrm{~km}$. In Sect. 5, the results are summarised and discussed.

\section{Set-up}

We use the MIT-gcm code, a finite-volume, nonlinear, nonhydrostatic numerical model which solves the equations of motion under the Boussinesq approximation (Marshall et al., 1997). We define a Cartesian co-ordinate system $(O, x, z)$ whose origin is at the upper western corner of the twodimensional rectangular domain. Although we allow for velocities in the meridional direction, the adjective "horizontal" will refer to the $x$ (zonal) direction. The reference stratification profile we consider is a typical profile observed in the Bay of Biscay in mid to late summer, when the thermocline is best developed and was used by e.g., Maugé and Gerkema (2008) and GSG10. This reference profile will be hereafter referred to as S1; the corresponding Brunt-Väisälä profile $N(z)$ is characterised by a strong seasonal thermocline which maximal value is $1.6 \times 10^{-2} \mathrm{rad}_{\mathrm{s}}^{-1}$ at $58 \mathrm{~m}$ depth. Local generation is observed during mid to late summer, i.e. when $\mathrm{S} 1$ is measured. For purposes that will become clear in Sect. 3, another stratification profile referred to as S2 will also be used, with a maximal value of $N(z)$ equal to $1.2 \times 10^{-2}$ rad.s $^{-1}$, corresponding to a late spring stratification. The Brunt-Väisälä profiles for $\mathrm{S} 1$ and $\mathrm{S} 2$ are displayed in Fig. 1.

We impose an ITB on the western boundary of the domain. The set-up of this beam is slightly different than in GSG10. In the first place, the analytical expression of the forcing envelope is the profile of an internal wave beam as found by Thomas and Stevenson (1972) in a non-rotating fluid of constant $N$ and extended to a rotating fluid by Peat (1978), namely:

$$
A(z)=\mathcal{A} \int_{-\infty}^{+\infty} k \exp \left(-\frac{1}{3}\left(\frac{\lambda_{0} k}{2 \pi}\right)^{3}+i k\left(z+\frac{H}{2}\right)\right) d k .
$$

$\mathcal{A}$ is a normalisation factor such that the maximal value of $|A(z)|$ is equal to $12 \mathrm{~cm} \cdot \mathrm{s}^{-1}$, which is approximately the maximal value of the along-beam velocity in the Bay of Biscay (e.g., Gerkema et al., 2004). $H=4370 \mathrm{~m}$ is the total depth of the domain and $\lambda_{0}$ a typical value for the vertical wavelength. We set $\lambda_{0}=1500 \mathrm{~m}$, which is representative of the ITB in the Bay of Biscay. The zonal, meridional and vertical velocities and the temperature at the western boundary (referred to as $u_{b}, v_{b}, w_{b}$ and $T_{b}$ respectively) are found through the polarisation relations:

$$
\begin{aligned}
& u_{b}(z, t)=\sqrt{\frac{N_{1 / 2}^{2}-\omega_{0}^{2}}{N_{1 / 2}^{2}-f^{2}}} \Im\left(A e^{i \omega_{0} t}\right), \\
& v_{b}(z, t)=\frac{f}{\omega_{0}} \sqrt{\frac{N_{1 / 2}^{2}-\omega_{0}^{2}}{N_{1 / 2}^{2}-f^{2}}} \Re\left(A e^{i \omega_{0} t}\right),
\end{aligned}
$$

$w_{b}(z, t)=\sqrt{\frac{\omega_{0}^{2}-f^{2}}{N_{1 / 2}^{2}-\omega_{0}^{2}}} u_{b}(z, t)$,

$T_{b}(z, t)=T_{0}(z)+\frac{N_{1 / 2}^{2}}{g \alpha \omega_{0}} \sqrt{\frac{\omega_{0}^{2}-f^{2}}{N_{1 / 2}^{2}-f^{2}}} \Re\left(A e^{i \omega_{0} t}\right)$,

with $N_{1 / 2}=1.315 \times 10^{-3}$ rad.s $^{-1}$ the Brunt-Väisälä frequency at mid-depth (which is the same for S1 and S2), $\omega_{0}$ the M2 tidal frequency in the Bay of Biscay corresponding to a period of 12.42 hours (hereafter referred to as the M2 period) and $f=1.04 \times 10^{-4} \mathrm{rad} . \mathrm{s}^{-1}$ the Coriolis parameter at $45^{\circ} \mathrm{N}$. We set $\alpha=1.89 \times 10^{-4} \mathrm{~K}^{-1}$ and therefore $T_{0}$ has to vary from $4^{\circ} \mathrm{C}$ to $17^{\circ} \mathrm{C}$ for $\mathrm{S} 1$ and to $14.5^{\circ} \mathrm{C}$ for $\mathrm{S} 2$. Finally, $\Re$ and $\Im$ denote the real and imaginary parts respectively.

The ISWs in the Bay of Biscay are about 1 to $2 \mathrm{~km}$ long in the direction of their propagation and have amplitudes up to $80 \mathrm{~m}$ (New and Pingree, 1990, 1992; New and da Silva, 2002). In order to resolve an ISW with 20 points in each direction, the horizontal grid spacing is set to $50 \mathrm{~m}$ and the vertical grid spacing is set to $4 \mathrm{~m}$ in the upper $170 \mathrm{~m}$. Such a high vertical resolution is not necessary everywhere and is therefore equal to $25 \mathrm{~m}$ in the lower $3900 \mathrm{~m}$. The transition between the two vertical resolutions is achieved as in GSG10 and is centred around $330 \mathrm{~m}$ depth. The grid size is $4000 \times 1 \times 234$ points, implying that the horizontal extent of the domain is $200 \mathrm{~km}$. At the eastern side of the domain, a sponge layer of length $50 \mathrm{~km}$ is implemented. It consists 
of adding to the momentum equations an extra term characterised by a relaxation timescale, relaxing the motions from the boundary of the sponge layer facing the interior of the domain (or inner boundary) to the value prescribed at the end of the domain. In order to avoid reflections at the inner boundary back to the interior of the domain, the relaxation time is progressively decreased from $12.42 \mathrm{~h}$ at the inner boundary to $12.42 \times 10^{-3} \mathrm{~h}$ at the boundary of the domain. This is the sponge layer implemented by default in the MIT-gcm which corresponds to the Herbaut formulation as described in Zhang and Marotzke (1999).

The background viscosity and thermal diffusivity are set, respectively, to $10^{-4} \mathrm{~m}^{2} \cdot \mathrm{s}^{-1}$ and $10^{-5} \mathrm{~m}^{2} \cdot \mathrm{s}^{-1}$ to reproduce the eddy diffusivities that are expected in the ITB in the Bay of Biscay. Considering $\max (|A(z)|)$ and $\lambda_{0}$ as reference velocities and length scales, respectively, the Reynolds number in the ITB is therefore in the order of $10^{6}$. To avoid accumulation of energy at the grid scale due to nonlinear energy transfers, a KPP scheme acting on the vertical viscosity and diffusivity is enabled (Large et al., 1994), which induces very high values of the vertical viscosity, up to $0.1 \mathrm{~m}^{2} . \mathrm{s}^{-1}$ where the beam impinges on the thermocline. In the horizontal direction, biharmonic viscosity and diffusivity are both set to $10^{3} \mathrm{~m}^{4} \cdot \mathrm{s}^{-1}$.

\section{Internal tidal beam impinging on a thermocline initially at rest}

\subsection{Generation of internal solitary waves}

Starting from rest, we carry out a numerical experiment hereafter referred to as experiment E1, using the stratification profile $\mathrm{S} 1$. E1 is run for $16 \mathrm{M} 2$ periods. We observe a strong scattering of the ITB after interaction with the thermocline, as described by Gerkema (2001). This suggests that we are in the "moderate regime" for the density jump, which favours local generation. This scattering can be seen in Fig. 2b. In Fig. 2a, very weak signatures of mode-2 ISWs are to be seen, which are approximately $2.5 \mathrm{~km}$ wide, with an induced isopycnal displacement typically smaller than $10 \mathrm{~m}$. This contradicts observations, for which $80 \mathrm{~m}$-amplitude, mode- 1 ISWs are observed for the stratification profile S1. However, there is some uncertainty on the parameters required for local generation to occur, which are the horizontal phase speed of the ITB and the stratification profile in the thermocline.

The sensitivity of the local generation process to the stratification profile is addressed in a second experiment, hereafter referred to as E2, which is run with stratification S2 for 16 periods: Fig. 3a shows that mode-1 ISWs are now generated at a rate of one train per $\mathrm{M} 2$ period, leading to a strong scattering of the ITB (Fig. 3b). We shall show below that these ISWs still do not compare favourably with in situ measurements in the Bay of Biscay, the distance between two wave trains and the ISWs amplitude being too small. Also, lo- (a)

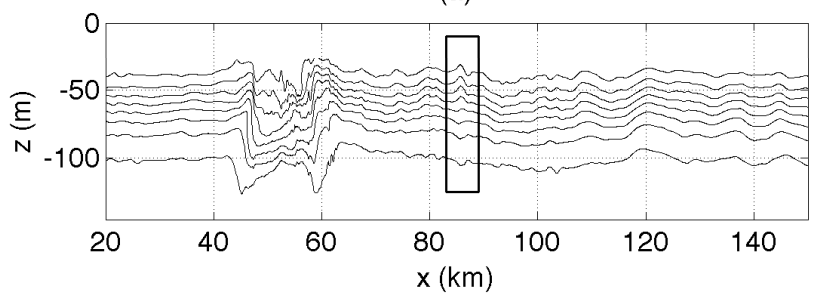

(b)

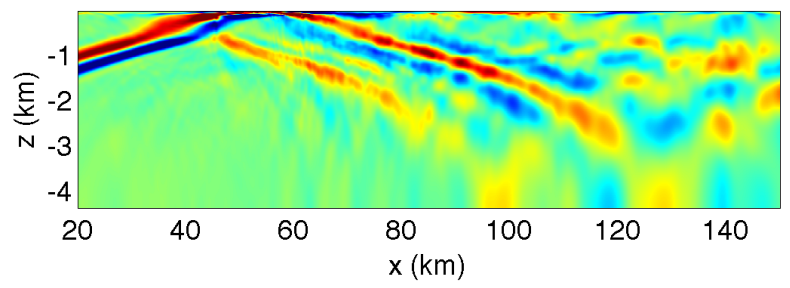

Fig. 2. Snapshot taken from E1, $16 \mathrm{M} 2$ periods after the start of the simulation. (a): Contours of a few isopycnals around the thermocline. A mode-2 ISW is framed. (b): Horizontal velocity field in the domain, the colour scale ranging from -12 (blue) to $+12 \mathrm{~cm} . \mathrm{s}^{-1}$ (red). Note that $x \geq 20 \mathrm{~km}$ and that, therefore, the forcing beam at the western boundary has already been refracted by the varying $N(z)$ profile.

(a)

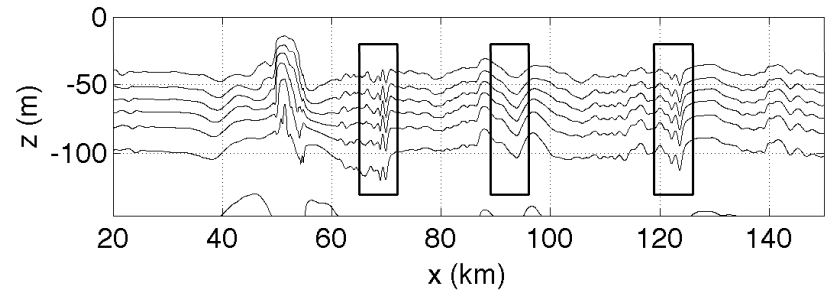

(b)

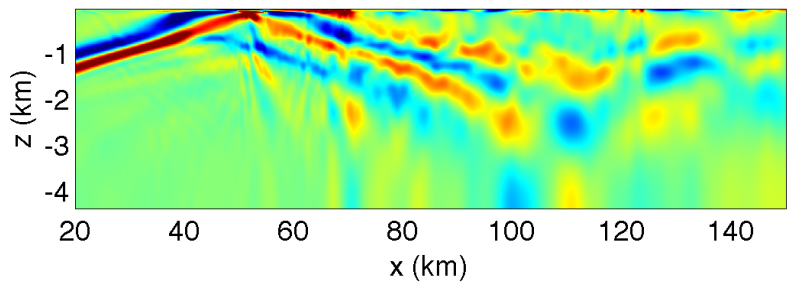

Fig. 3. Caption as for Fig. 2, but for E2, 11.5 M2 periods after the start of the simulation. The three main wave trains are framed in (a) and have all been generated one M2 period after each other.

cal generation is not known to happen in late spring, during which a stratification profile like S2 is expected to be found. This implies that some extra process may come into play in the local generation of ISWs, an important point further addressed in Sect. 4.

Figure 4 displays the temporal evolution of the isopycnal in E2, where $N(z)$ is maximal, over one M2 period. The leading deformations of the ISW trains, each of them 


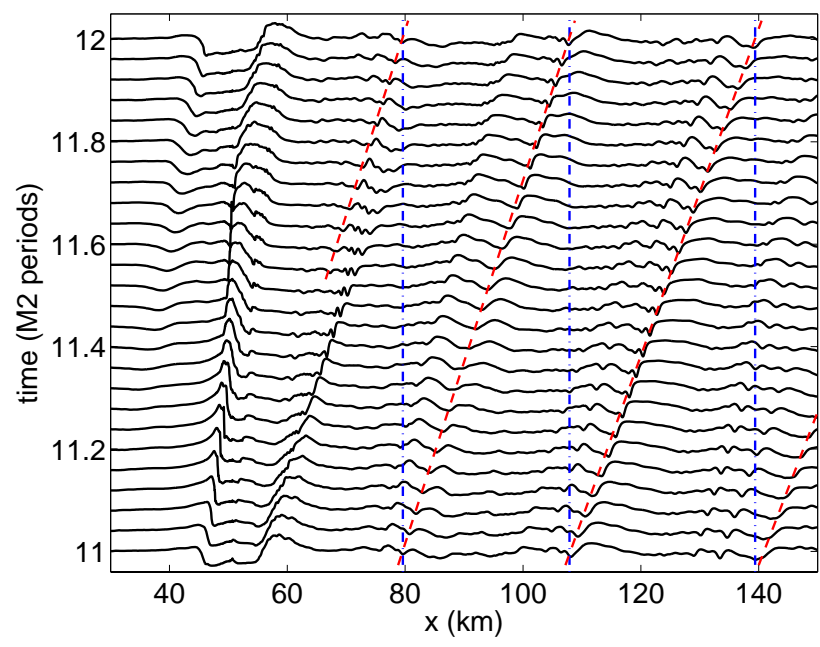

Fig. 4. In E2, vertical displacement of the isopycnal where $N(z)$ is maximal between 11 and $12 \mathrm{M} 2$ periods after the start of the simulation. Two isopycnals on the left side of the pictures are separated by $1 / 25 \mathrm{M} 2$ periods, also corresponding to $30 \mathrm{~m}$ in terms of displacement amplitude. Red, dashed lines follow four different wave trains at different locations, each of them having been generated one M2 period after the other. The blue, dash-dotted lines help noticing that the four leading deformations highlighted by the red, dashed lines can be seen as four different life stages of a single wave train. The large displacement located between 40 and $60 \mathrm{~km}$ corresponds to the ITB impact on the thermocline.

having been generated one M2 period after the other, are also tracked. We chose to display this temporal evolution in a rather early stage of the simulation as high frequency motions tend to increase and make the pictures harder to interpret. However, the main features of the ISWs remain the same over the course of the simulation. Figure 4 shows that the induced isopycnal displacement is in the order of $25 \mathrm{~m}$, while the observations of New and Pingree $(1990,1992)$ report values up to $80 \mathrm{~m}$, as mentioned above.

The distance between two trains of ISWs needs also to be discussed. Regardless of the location of where the ITB impinges on the thermocline, the internal tide in the Bay of Biscay is dominated in the upper $100 \mathrm{~m}$ by a signal of horizontal wavelength $50 \mathrm{~km}$ (e.g., Pingree and New, 1991; Pichon and Correard, 2006). We will refer to this signal as the "upper tide", whose physical features are discussed in Gerkema et al. ( $\$ 42004)$. This signal can be qualitatively described as a background oscillation of the thermocline. In addition, the distance between two trains of ISWs has also been measured to be about $50 \mathrm{~km}$ (e.g., New and da Silva, 2002), suggesting a connexion between these two types of waves. In E2, by contrast, two trains of ISWs are rather separated by 25 to $30 \mathrm{~km}$ (see Figs. 3a and 4), meaning that these ISWs propagate at almost half the speed as ISWs observed in the Bay of Biscay.

\subsection{Interpretation of experiments E1 and E2}

GSG10 proposed two different selection conditions to predict what mode of ISWs will be selected, if any. Although the two conditions were qualitatively insightful, the "far-field condition" proved to give better quantitative results when addressing the problem of a realistic stratification, as is the case here. Hence, we will focus on this condition only.

The "far-field condition" relies on the fact that ISWs evolve from internal waves that are trapped in the thermocline, i.e. whose frequencies are comprised between $N_{0}$ and the maximum value of $N(z)$. In that case, a forcing beam of horizontal phase speed close to the phase speed of the desired trapped mode will select that mode, provided that the amplitude of the forcing beam is large enough to promote nonlinear effects. Increasing the density jump or decreasing the horizontal phase speed of the beam has the effect of selecting ISWs of higher modes.

Since the ITB frequency is the M2 frequency, the horizontal phase speed of the ITB (hereafter referred to as $v_{\phi}$ ) is estimated by measuring the dominant horizontal wavelength of the ITB. Note also that since a wave train is created every M2 periods at the beam impact location, the phasematching condition implies that the distance between two wave trains matches the horizontal wavelength of the ITB. Figure $2 b$ shows that the horizontal wavelength of the ITB is approximately $18 \mathrm{~km}$ which gives a value for $v_{\phi}$ of about $0.4 \mathrm{~m} . \mathrm{s}^{-1}$. The phase speeds of the trapped modes are computed via a normal mode approach, using the stratification profile $N(z)$. Searching for a vertical velocity solution of the linear inviscid Boussinesq equations of the form $w(x, z, t)=$ $W(z) e^{i(K x-\Omega t)}(K>0$ for eastward-propagating waves and $\Omega$ not necessarily equal to $\omega_{0}$ ), we solve the Taylor-Goldstein equation, along with boundary conditions consisting of a flat bottom and a rigid lid:

$\frac{d^{2} W}{d z^{2}}+K^{2} \frac{N^{2}(z)-\Omega^{2}}{\Omega^{2}-f^{2}} W(z)=0$,

$W(z=-H)=W(z=0)=0$.

The system of Eqs. (7) and (8) has an infinite sequence of eigenfunctions $W_{n}$ associated with eigenvalues $K_{n}$ ( $n$ being the mode number) yielding the phase speed of each mode $c_{n}=\Omega / K_{n}$.

The phase speeds of the two first modes and of the forcing ITB are displayed in Fig. 5 for S1 and in Fig. 6 for S2. Two regimes are to be distinguished. The first one is referred to as the "free" regime and is associated with frequencies low enough for plane waves to be able to propagate below the seasonal thermocline. The second regime is referred to as the "trapped" regime (coloured in grey in Figs. 5 and 6), in which frequencies are typically higher than the Brunt-Väisälä frequency outside the seasonal thermocline, implying that plane waves of such frequencies could not propagate beneath the thermocline. In this regime, the phase speed of the waves 


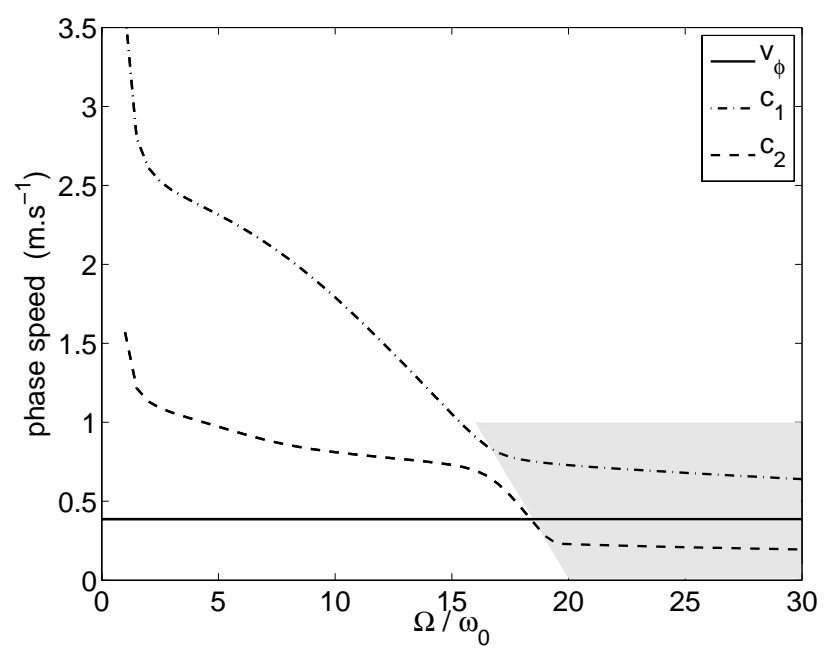

Fig. 5. Phase speeds of the two first internal modes versus their frequency for stratification S1 and comparison with the horizontal phase speed of the ITB. The "trapped regime" is the grey-shaded area (adapted from GSG10).

weakly varies with frequency, as opposed to the free regime. We will consider this phase speed as constant and call it the "trapped phase speed". This phase speed has to match $v_{\phi}$ for the corresponding mode to be forced. Figure 5 compares $v_{\phi}$ with the phase speeds of the two first modes versus frequency for $\mathrm{S} 1$. In this case, $v_{\phi}$ lies between these two phase speeds so that no trapped motion can be efficiently forced in the seasonal thermocline. This could explain why no strong signature of ISWs is detected in E1. For S2 however, the trapped phase speeds of the modes are lower due to lower values of the Brunt-Väisälä frequency in the seasonal thermocline. The trapped phase speed of the first mode is then brought closer to $v_{\phi}$, hence the observation of mode-1 ISWs, as seen in Fig. 6.

To conclude the comparison between E1 and E2, E1 is an experiment set up with parameters for which local generation is known to be favoured. However, local generation is not convincingly observed. To examine the sensitivity of the local generation process to the stratification profile, a second experiment E2 is designed, with the stratification profile slightly departing from the reference situation on which E1 was based. Mode-1 ISWs are now locally generated but their amplitude and the distance between two trains of ISWs noticeably differ with observations in the Bay of Biscay. We will see in the next section that a set-up, including more components of the internal tide and especially the background motion of the pycnocline, can reproduce observations more accurately. This is in contrast with all previous papers (including GSG10), in which the problem of an ITB impinging on a thermocline initially at rest was considered.

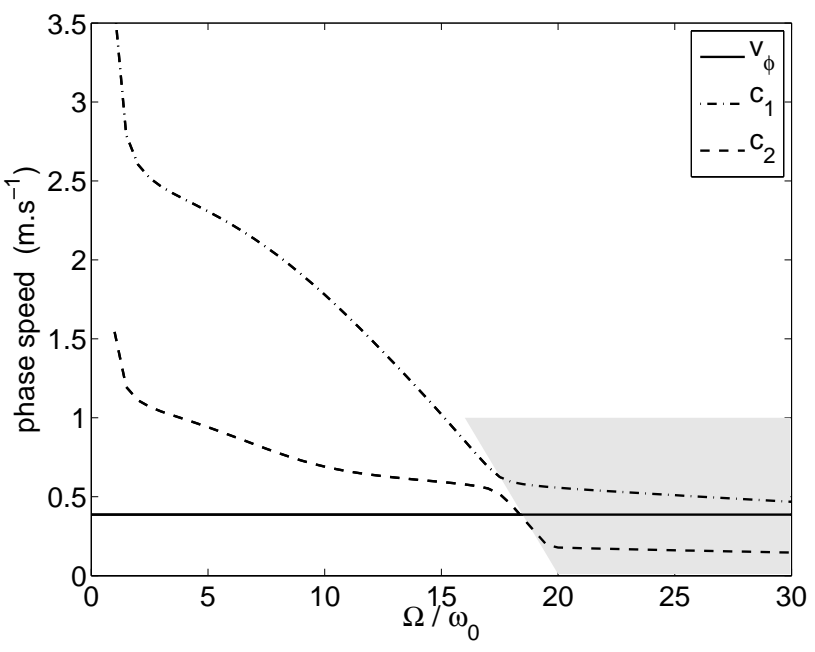

Fig. 6. Caption as for Fig. 5, for stratification S2.

\section{A more realistic configuration}

As we observed in Sect. 3.1, the trains of ISWs generated in E2 are separated by distances which are selected by the wavelength of the forcing beam, whereas in the ocean these trains are separated by a distance approximately equal to the wavelength of the upper tide, regardless of the position and characteristics of the ITB. We present here a way to set up a numerical experiment, complex enough to model a more realistic forcing, yet numerically affordable as there is no need to include the continental shelf and slope. Indeed, to include this bathymetry would imply a doubling of the size of the domain in the horizontal direction, with high horizontal resolution needed throughout most of the domain, especially near the shelf break. Such a complete bathymetry would help retrieving a more realistic ITB as well as the upper tide but is numerically expensive. We then decided to proceed in two steps, bearing some similarities with a nested grid approach.

First, a low-resolution, low-amplitude numerical experiment, hereafter referred to as $\hat{E} 3$ and including a continental shelf is designed. It is noticeably different from E1 and E2 and has a much reduced numerical cost. The length of the domain is $400 \mathrm{~km}$, the vertical and horizontal grid resolutions are respectively $25 \mathrm{~m}$ and $400 \mathrm{~m}$. The bathymetry has been designed by Gerkema et al. (2004) from measurements in the Bay of Biscay and can be seen in Fig. 7. The continental shelf and the deep ocean are both made flat and respectively 100 and $216 \mathrm{~km}$ long. The stratification profile S1 is used. Two sponge layers at each end of the domain are implemented. They have the same characteristics as in E1 and E2, except for their length which is now $100 \mathrm{~km}$. The viscosities, thermal diffusivities and biharmonic viscosity remain unchanged and the KPP scheme is turned off as it is now unnecessary. The forcing is barotropic, with an amplitude of the horizontal velocity being equal to $0.1 \mathrm{~mm} \cdot \mathrm{s}^{-1}$ on the eastern boundary 
(a)

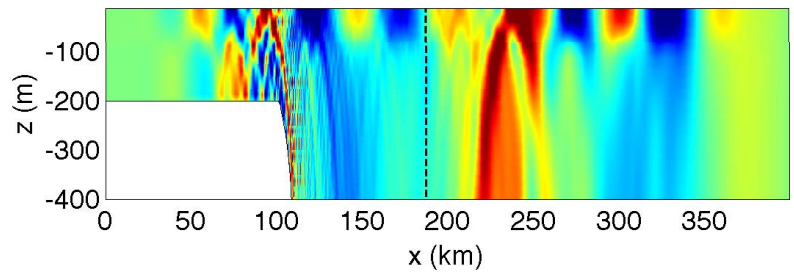

(b)

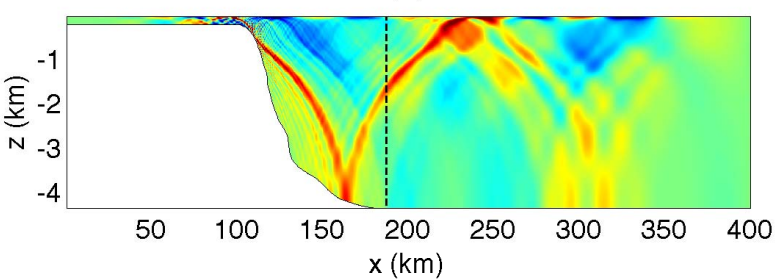

Fig. 7. Snapshot taken from experiment Ê3, $30 \mathrm{M} 2$ periods after the start of the simulation. (a): Close-up of the horizontal velocity field in the upper $400 \mathrm{~m}$ of the domain. (b): horizontal velocity field in the whole domain, the colour scale ranging from -1 (blue) to $+1 \mathrm{~mm} \cdot \mathrm{s}^{-1}$ (red). The white region is the bathymetry and the dashed line is the line from where the tidal forcing is extracted.

(facing the open ocean in our case). A barotropic flow is also imposed on the western boundary in order to satisfy flux conservation.

The amplitude of the eastern forcing is about a hundred times less than in the Bay of Biscay to retrieve a dynamics as linear as possible and reduce the computational cost. Indeed, the large-scale structure of the internal tide we wish to model is governed by a quasi-linear dynamics. Nonlinearities become important when the ITB interacts with the thermocline and are associated with processes which cannot occur in this low-resolution experiment. The experiment is run for $30 \mathrm{M} 2$ periods with the same time step as in E1 and E2. Harmonic analysis of the velocity and temperature fields is then performed at $\omega_{0}$ on the twelve last M2 periods, during which the permanent regime is established.

Second, a numerical experiment E3 is designed. We choose to use the stratification S1 again as in E1. E3 is almost identical to E1 except for the forcing. Indeed, instead of using the analytical expression $A(z)$ (Eq. 2) for the ITB, the result of the harmonic analysis of $\hat{E} 3$ is extracted at location $x=188 \mathrm{~km}$ (see Fig. 7). This gives complex vertical profiles for the forcing velocity and temperature fields which are applied directly at the western boundary. The amplitude of this forcing is also rescaled such that the maximal alongbeam velocity is equal to $17 \mathrm{~cm} . \mathrm{s}^{-1}$ at the boundary, which ensures that the amplitude of the ITB is the same as in E1 at depth $z=-1400 \mathrm{~m}$. For purposes of illustration, the real and imaginary part of the horizontal velocity profile are displayed in Fig. 8. As this forcing includes a significant barotropic component, a barotropic flow is set at the eastern boundary that preserves the flux through the whole domain. (a)

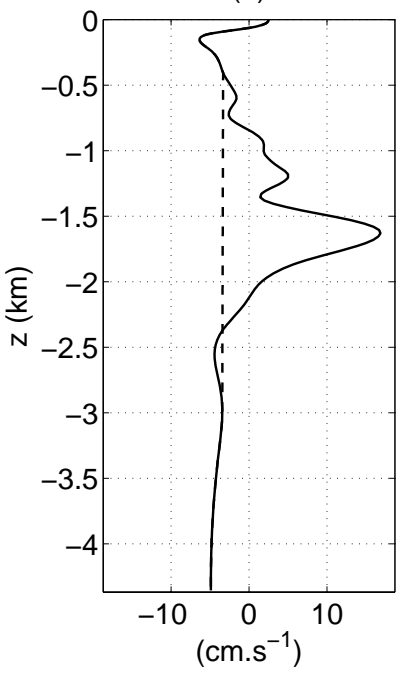

(b)

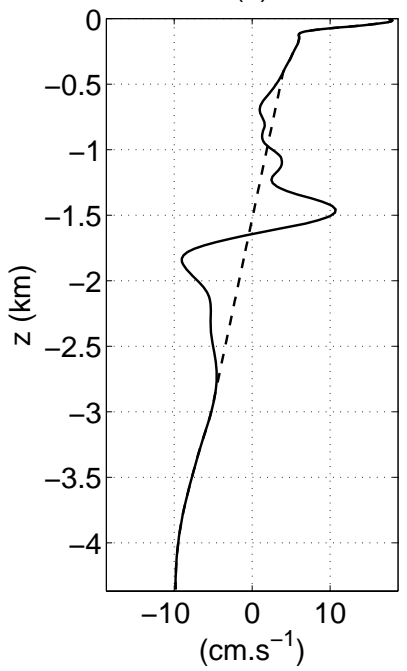

Fig. 8. Real (a) and imaginary (b) parts of the horizontal velocity forcing for E3 (solid line) and E4 (dashed line).

(a)

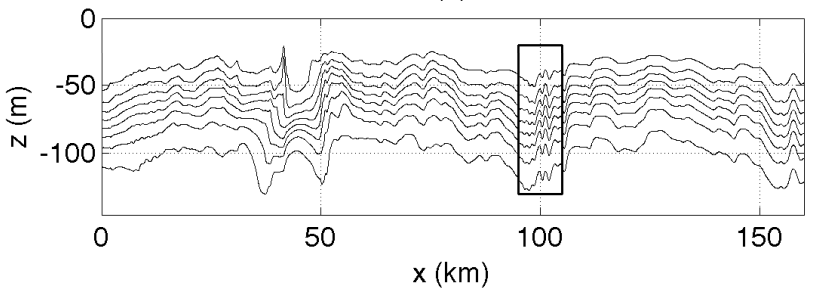

(b)

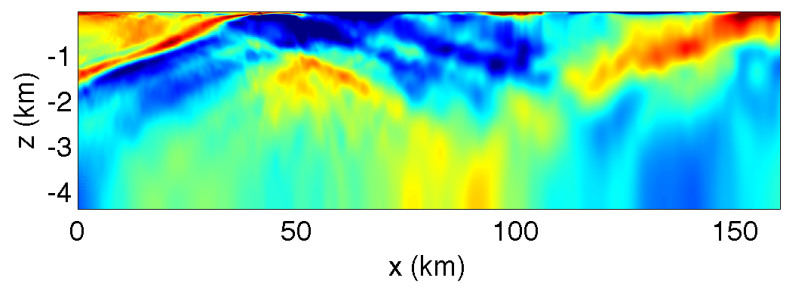

Fig. 9. Caption as for Fig. 2, this time for E3, $12 \mathrm{M} 2$ periods after the start of the simulation.

Figure 9 shows a snapshot of the wave field, 12 M2 periods after the start of the simulation and Fig. 10 shows the evolution of the isopycnal where $N(z)$ is maximum between 11 and $12 \mathrm{M} 2$ periods after the start of the simulation. Unlike in E1 and as in E2, mode-1 ISWs can be seen. Their amplitude is nearly the same as in E2. Yet, the stratification used here is S1, as in E1 where no mode-1 ISWs were observed. This cannot be explained by a higher value of the horizontal phase speed of the ITB, as its wavelength is smaller than in E1 $(\approx 15 \mathrm{~km})$. From GSG10 predictions (see Fig. 5), the sole ITB would rather force mode-2 ISWs. 


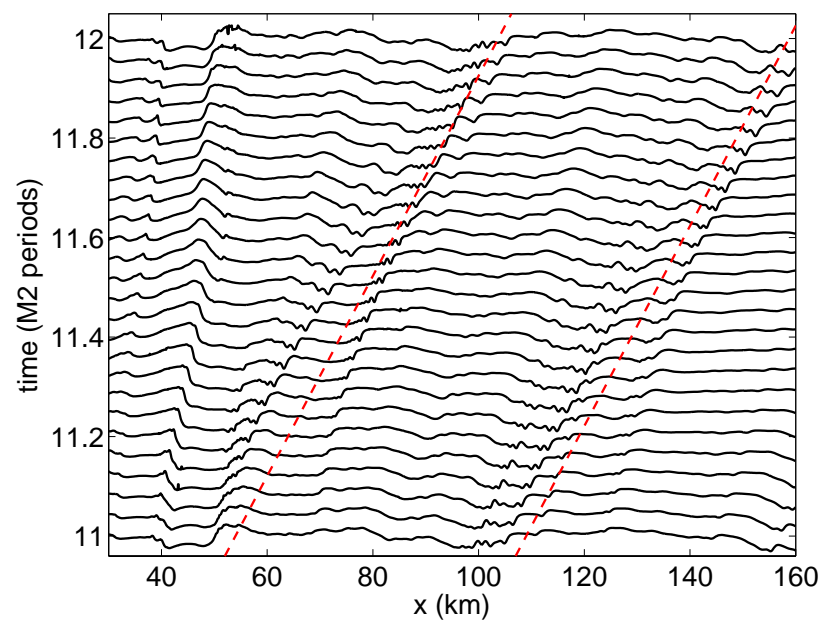

Fig. 10. Caption as for Fig. 4, this time for E3. The two dashed, red lines are now separated by $55 \mathrm{~km}$ and correspond to velocities of $1.23 \mathrm{~m} . \mathrm{s}^{-1}$ (namely, $55 \mathrm{~km}$ per M2 period). The large displacement located between 40 and $55 \mathrm{~km}$ corresponds to the ITB impact on the thermocline.

It can be seen on Fig. 10 that the trains of ISWs in E3 are now separated by $55 \mathrm{~km}$, meaning that they propagate approximately twice as fast as the ISWs observed in E2 and are consistent with the observations in the Bay of Biscay. The ISWs observed in E3 could however be due to the upper tide experiencing nonlinear steepening and nonhydrostatic evolution into ISWs, as suggested by New and Pingree (2000). In this case, ISWs would be generated even without an ITB. To confirm that it is not the case, a last experiment E4 is designed, based on E3 except that the ITB is "removed" from the forcing field by linearly interpolating this forcing between its values at depths $z=-408 \mathrm{~m}$ and $z=-2980 \mathrm{~m}$ and keeping it unchanged everywhere else (see Fig. 8). Figures 11 and 12 show that, unlike E3, E4 displays no strong nonlinear features in the thermocline. Figure 12 indicates also that the carrier wave of the ISWs in E3 can be superposed on the interfacial displacement in E4, which is the upper tide. It therefore seems that the local generation mechanism is very sensitive to the presence of a background wave field in the upper $100 \mathrm{~m}$.

\section{Conclusion and discussion}

We have presented two-dimensional numerical experiments using parameters representative of the Bay of Biscay when local generation is observed. The first two experiments address the problem of an ITB impinging on a thermocline initially at rest. The first experiment E1, using a mid to late summer stratification that is typical of the Bay of Biscay when local generation is observed and a realistic ITB, does not lead to a convincing generation of ISWs, thus disagreeing with the observations. The second experiment E2 (a)

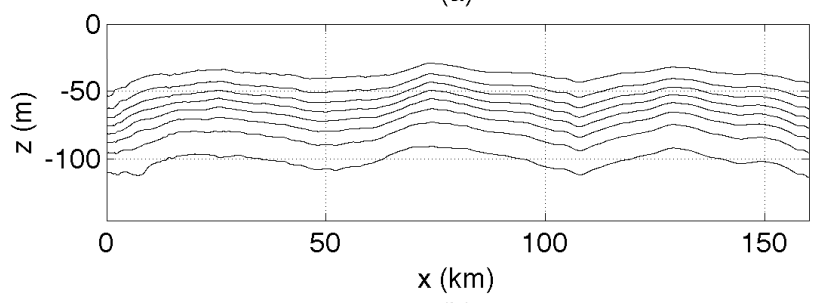

(b)

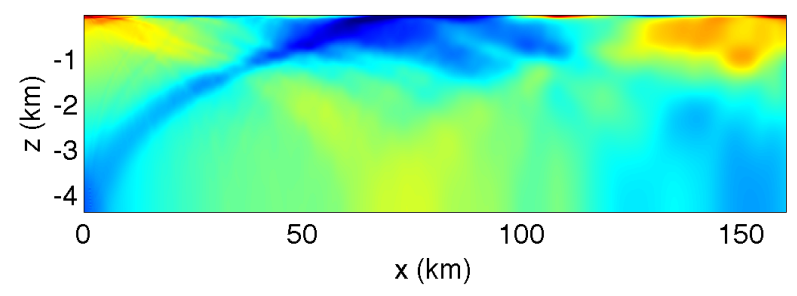

Fig. 11. Caption as for Fig. 9, for E4.

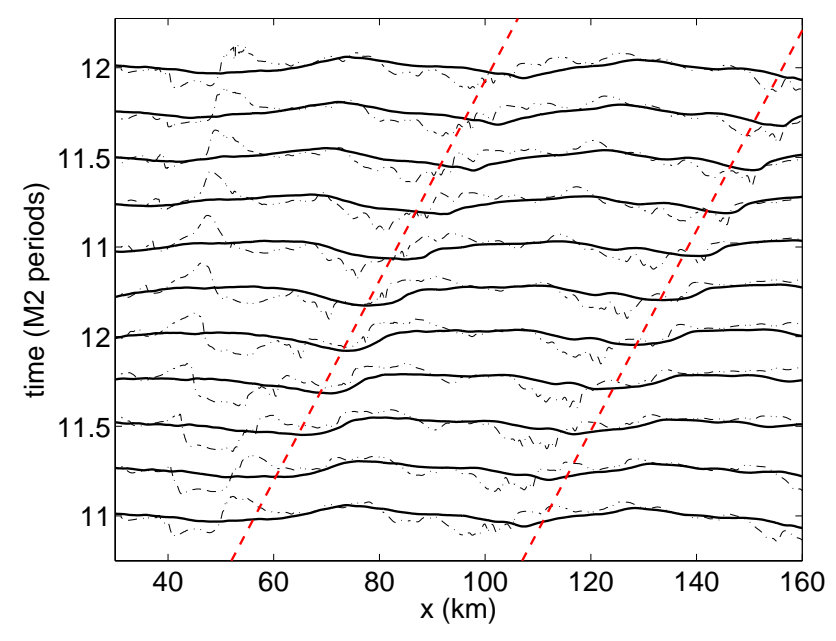

Fig. 12. Caption as for Fig. 4, except that two isopycnals on the left side of the pictures are separated by $1 / 10 \mathrm{M} 2$ periods and also by $40 \mathrm{~m}$ in terms of displacement amplitude. Solid lines refer to E4, dash-dotted lines to E3.

uses a slightly reduced density jump across the seasonal thermocline similar to that observed in late spring. In this case mode-1 ISWs are generated. The vertical structures (mode numbers) of the ISWs developing in E1 and E2, if any, agree with the predictions of the far-field condition derived in GSG10. Nonetheless, the distance between two wave trains in E2 displays significant discrepancies with the observations. In the Bay of Biscay indeed, this distance is about $50 \mathrm{~km}$, which is also the dominant horizontal wavelength of the internal tide in the upper $100 \mathrm{~m}$ (referred to as the upper tide), regardless of the characteristics of the ITB. In E2 by contrast, the ISW trains are separated by 25 to $30 \mathrm{~km}$, close enough to $18 \mathrm{~km}$, the horizontal wavelength of the forcing ITB, for it to generate mode-1 ISWs. 
A possible explanation for this discrepancy relies upon the interaction of the ITB with the upper tide generated at the shelf break and propagating throughout the domain. A third numerical experiment E3 was thus designed, forced by the internal tide generated by a barotropic flow on the continental slope. The stratification is the same as in E1 (and as, therefore, in the Bay of Biscay when local generation occurs). This experiment shows that mode-1 ISWs are generated, each train being now separated by about $55 \mathrm{~km}$, in agreement with observations. To investigate the role of the upper tide in the generation process of these ISWs, a fourth and last experiment $\mathrm{E} 4$ was designed, which forcing includes the upper tide but not the ITB. No ISWs appear but the interfacial displacement can be superposed on the carrier wave of the ISWs observed in the third experiment.

To sum up, E1 and E2 show that an ITB impinging on a seasonal thermocline can generate ISWs but that the mechanism is very sensitive to the amplitude of the density jump across the seasonal thermocline, in agreement with GSG10 as well as the predictions of previous works (Gerkema, 2001; Akylas et al., 2007). E2 also shows that mode-1 ISWs are generated but with quantitatively different features than those of the ISWs observed in the Bay of Biscay. On the other hand, the upper tide alone does not evolve into trains of ISWs. Only when an ITB interacts with the upper tide is the formation of ISWs observed, with physical characteristics close to the observations. The propagation speeds and distance between trains of ISWs are now controlled by the upper tide but the ITB supplies a significant part of the energy necessary for the generation of ISWs, provided that the stratification allows for an efficient interaction of that beam with the thermocline. This mechanism is quite different than the classical image of the local generation process and could actually be invoked in many places of the world where ISWs are observed.

Conjectures on the mechanism of how energy is transferred to the thermocline in the presence of the upper tide can be proposed. For instance, the upper tide can be modelled as an oscillating horizontal shear flow. As this oscillating motion is synchronous with those induced in the thermocline by the ITB, the phase shift between the ITB and the upper tide may be such that $c_{1}$ would be reduced by Doppler effect at the location of the impact of the ITB and hence brought closer to $v_{\phi}$. If it is brought close enough, the selection condition discussed about in $\$ 3.2$ would predict that mode- 1 ISWs are generated. Further analytical work is required to address the validity of this conjecture.

We are quite aware that several effects have been neglected. First, our numerical experiments are twodimensional. Also, Gerkema et al. (2008) showed that the ITB in the Bay of Biscay is very sensitive to non-traditional effects. Including these effects would modify the initialisation of experiments E3 and E4. Also, the relative amplitudes of the ITB and of the upper tide might be different than those set in E3. Performing three-dimensional realistic non-hydrostatic (and nonlinear) numerical experiments of the Bay of Biscay, still a computational challenge nowadays, appears to be necessary to assess the respective roles of the ITB and the upper tide in the generation of ISWs and in the control of their spatial structure.

Acknowledgements. N.G. is supported by a grant from the Délégation Générale de l'Armement. This work has been supported by ANR contract PIWO (\# 08-BLAN-0113). Numerical experiments were performed on the French supercomputer center IDRIS, through contracts 080580 and 090580 . We thank Yves Morel and Annick Pichon for sharing their knowledge about the Bay of Biscay.

Edited by: R. Grimshaw

Reviewed by: two anonymous referees

\section{References}

Akylas, T. R., Grimshaw, R. H. J., Clarke, S. R., and Tabaei, A.: Reflecting tidal wave beams and local generation of solitary waves in the ocean thermocline, J. Fluid Mech., 593, 297-313, 2007.

Azevedo, A., da Silva, J. C. B., and New, A. L.: On the generation and propagation of internal solitary waves in the southern Bay of Biscay, Deep-Sea Res. Pt. I, 53, 927-941, doi:10.1016/j.dsr. 2006.01.013, 2006.

da Silva, J. C. B., New, A. L., Srokosz, M. A., and Smyth, T. J.: On the observability of internal tidal waves in remotelysensed ocean colour data, Geophys. Res. Lett., 29(12), 15691572, doi:10.1029/2001GL013888, 2002.

da Silva, J. C. B., New, A. L., and Azevedo, A.: On the Role of SAR for observing "local generation" of internal solitary waves off the Iberian Peninsula, Can. J. Remote Sens., 33, 388-403, 2007.

da Silva, J. C. B., New, A. L. M., and Magalhaes, J. M.: Internal Solitary Waves in the Mozambique Channel: Observations and Interpretation, J. Geophys. Res., 114, C05001, doi: 10.1029/2008JC005125, 2009.

Delisi, D. P. and Orlanski, I.: On the role of density jumps in the reflexion and breaking of internal gravity waves, J. Fluid Mech., 69, 445-464, doi:10.1017/S0022112075001516, 1975.

Gerkema, T.: Internal and interfacial tides: Beam scattering and local generation of solitary waves, J. Mar. Res., 59, 227-255, 2001.

Gerkema, T., Lam, F.-P. A., and Maas, L. R. M.: Internal tides in the Bay of Biscay: Conversion rates and seasonal effects, Deep-Sea Res. Pt. II, 51, 2995-3008, 2004.

Gerkema, T., Zimmerman, J. T. F., Maas, L. R. M., and van Haren, H.: Geophysical and Astrophysical Fluid Dynamics Beyond the Traditional Approximation, Rev. Geophys., 46, RG2004, doi:10. 1029/2006RG000220, 2008.

Gill, A. E.: Atmosphere-Ocean Dynamics, Academic Press, 662 pp., 1982.

Grisouard, N., Staquet, C., and Gerkema, T.: Generation of internal solitary waves in a pycnocline by an internal wave beam: a numerical study, in review, J. Fluid Mech., 2010.

Helfrich, K. R. and Melville, W. K.: Long Nonlinear Internal Waves, Annu. Rev. Fluid Mech., 38, 395-425, doi:10.1146/ annurev.fluid.38.050304.092129, 2006. 
Konyaev, K., Sabinin, K., and Serebryany, A.: Large-amplitude internal waves at the Mascarene Ridge in the Indian Ocean, Deep-Sea Res. Pt. I, 42, 2075-2081, doi:10.1016/0967-0637(95) 00067-4, 1995.

Large, W. G., McWilliams, J. C., and Doney, S. C.: Oceanic vertical mixing: a review and a model with a nonlocal boundary layer parameterization, Rev. Geophys., 32, 363-404, doi: 10.1029/94RG01872, 1994.

Marshall, J., Adcroft, A., Hill, C., Perelman, L., and Heisey, C.: A finite-volume, incompressible Navier Stokes model for studies of the ocean on parallel computers, J. Geophys. Res., 102, 57535766, doi:10.1029/96JC02775, 1997.

Maugé, R. and Gerkema, T.: Generation of weakly nonlinear nonhydrostatic internal tides over large topography: a multimodal approach, Nonlin. Processes Geophys., 15, 233-244, doi:10.5194/npg-15-233-2008, 2008.

Munk, W. and Wunsch, C.: Abyssal recipes II: energetics of tidal and wind mixing, Deep-Sea Res. Pt. I, 45, 1977-2010, 1998.

New, A. L. and da Silva, J. C. B.: Remote-sensing evidence for the local generation of internal soliton packets in the central Bay of Biscay, Deep-Sea Res. Pt. I, 49, 915-934, 2002.

New, A. L. and Pingree, R. D.: Large-amplitude internal soliton packets in the central Bay of Biscay, Deep-Sea Res., 37, 513524, 1990.

New, A. L. and Pingree, R. D.: Local generation of internal soliton packets in the central Bay of Biscay, Deep-Sea Res., 39, 15211534, 1992.
New, A. L. and Pingree, R. D.: An intercomparison of internal solitary waves in the Bay of Biscay and resulting from Korteweg-de Vries-type theory, Prog. Oceanogr., 45, 1-38, 2000.

Ostrovsky, L. A. and Stepanyants, Y. A.: Do internal solitons exist in the ocean?, Rev. Geophys., 27, 293-310, doi:10.1029/ RG027i003p00293, 1989.

Peat, K. S.: Internal and inertial waves in a viscous rotating stratified fluid, Appl. Sci. Res., 33, 481-499, 1978.

Pichon, A. and Correard, S.: Internal tides modelling in the Bay of Biscay. Comparison with observations., Sci. Mar., 70S1, 65-88, 2006.

Pingree, R. D. and New, A. L.: Abyssal Penetration and Bottom Reflection of Internal Tidal Energy in the Bay of Biscay, J. Phys. Oceanogr., 21, 28-39, doi:10.1175/1520-0485(1991)021, 1991.

Thomas, N. H. and Stevenson, T. N.: A similarity solution for viscous internal waves, J. Fluid Mech., 54, 495-506, 1972.

Thorpe, S. A.: Nonlinear Reflection of Internal Waves at a Density Discontinuity at the Base of the Mixed Layer, J. Phys. Oceanogr., 28, 1853-1860, doi:10.1175/1520-0485(1998)028, 1998.

Wunsch, C. and Ferrari, R.: Vertical Mixing, Energy, and the General Circulation of the Oceans, Annu. Rev. Fluid Mech., 36, 281314, doi:10.1146/annurev.fluid.36.050802.122121, 2004.

Zhang, K. Q. and Marotzke, J.: The importance of openboundary estimation for an Indian Ocean GCM-data synthesis, J. Mar. Res., 57, 305-334, doi:doi:10.1357/002224099321618236, 1999. 